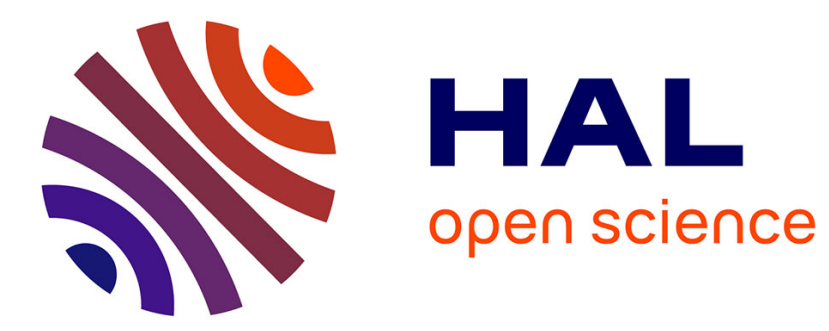

\title{
Les collections pour la recherche scientifique du XXI e siècle et au-delà
}

Roseli Pellens

\section{To cite this version:}

Roseli Pellens. Les collections pour la recherche scientifique du XXI e siècle et au-delà. Les collections naturalistes dans la science du XXIe siècle: une ressource durable pour la science ouverte 2021, pp.359372, 2021. hal-03409151

\section{HAL Id: hal-03409151 \\ https://hal.science/hal-03409151}

Submitted on 29 Oct 2021

HAL is a multi-disciplinary open access archive for the deposit and dissemination of scientific research documents, whether they are published or not. The documents may come from teaching and research institutions in France or abroad, or from public or private research centers.
L'archive ouverte pluridisciplinaire HAL, est destinée au dépôt et à la diffusion de documents scientifiques de niveau recherche, publiés ou non, émanant des établissements d'enseignement et de recherche français ou étrangers, des laboratoires publics ou privés. 


\title{
Chapitre 21
}

\section{Les collections pour la recherche scientifique du $\mathrm{XXI}^{\mathrm{e}}$ siècle et au-delà}

\author{
Roseli PeLlenS \\ Institut de Systématique, Évolution, et Biodiversité, Muséum national d'Histoire naturelle, \\ CNRS, Sorbonne Université, EPHE, UA, Paris, France
}

\subsection{Les collections dans la quête de connaissance}

Les études présentées dans ce volume montrent combien les collections accompagnent l'Humanité dans la quête de connaissance sur son histoire et son environnement. Les collections étaient plutôt focalisées sur la découverte de l'exotique, du précieux ou du commercial, dans une optique de répertorier et de cataloguer. Elles sont aujourd'hui la source des nouveaux regards que nous portons sur le vivant et sur notre place dans l'Univers. Les collections représentent aussi une base matérielle - une vision ramassée de la biodiversité - qui nous confronte avec le monde qui nous entoure. Nous pouvons difficilement imaginer l'émergence de plusieurs concepts dans la biologie moderne sans les nombreux retours, dépôts et examens de spécimens. Par exemple, dans le Voyage du Beagle, Charles Darwin rend bien visible les examens et les comparaisons qui lui ont permis de trouver de nombreux exemples pour développer la théorie de la sélection naturelle (Darwin 1845, 1859). À force de comparer, de revoir les mêmes spécimens, de définir les critères que les rassemblent, nous nous rendons compte de l'existence de l'éventail des possibles au sujet de l'émergence de cette diversité.

Le présent ouvrage nous montre qu'à force de travailler sur les collections, nous pouvons voir émerger d'autres informations et d'autres théories restées jusqu'à présent informulées ou imperceptibles. Les traditions de la science ouverte inhérentes à l'histoire de la systématique continuent à dessiner le tracé de ce chemin. 
Des nouvelles communautés de scientifiques découvrent cette ressource et s'imprègnent de ce savoir-faire et de cette modalité de retour sur les données.

\subsection{Trois grandes sortes de nouvelles utilisations des collections}

Ces études suggèrent l'existence de trois sortes majeures de nouvelles utilisations des collections.

\subsubsection{L'enrichissement des sciences du vivant, de l'Homme et de l'Univers avec les nouvelles technologies}

La première sorte d'utilisations rassemble les recherches qui utilisent des nouvelles technologies pour poursuivre la découverte du vivant, de l'Homme, des roches, des météorites et des minéraux. Ces recherches représentent, à différents degrés, le développement contemporain de la systématique, de la biologie évolutive, de l'anatomie comparée, de la minéralogie et de la géologie. L'innovation réside dans la capacité nouvelle à étudier des spécimens qui n'auraient pas pu être analysés sans les nouvelles technologies. L'accès à l'ADN, promu par les nouvelles technologies de séquençage (chapitre 13), et l'utilisation de la fluorescence vertejaune pour étudier les crustacés fossiles (chapitre 7) sont de bons exemples de ces développements. Les nouvelles informations obtenues sont non seulement moléculaires mais aussi anatomiques (Giribet 2010). Grâce à ces nouvelles technologies et après plus d'un siècle de controverses, l'existence d'une nervure additionnelle sur les ailes des insectes a pu ainsi être confirmée (Schubnel et al. 2020). De même pour l'étude de la diversité des girafes qui a pu être résolue, grâce, entre autres, au séquençage des spécimens de collections appartenant à des populations éteintes (Petzold et al. 2020 ; Petzold et Hassanin 2020). Les chapitres 3 , 4 et 5 nous offrent également des exemples de la manière dont ces nouvelles approches enrichissent plusieurs domaines scientifiques.

\subsubsection{Un réservoir d'informations sur l'environnement}

Le deuxième type d'utilisations intègre des recherches destinées à préciser et/ou affiner la connaissance des caractéristiques des environnements passés. Leur but est évidemment de comprendre les impacts des changements de l'environnement sur les organismes, principalement suite aux actions humaines. La valeur des teneurs chimiques ou des paramètres physiques peut être analysée à titre de proxys de l'environnement. L'occurrence de parasites, commensaux et symbiotes peut être étudiée parce qu'elle informe sur l'origine et les voies de transmission des 
pathogènes ravageant les cultures ou celles des maladies affectant l'Humanité. L'information peut être directement dans les tissus des spécimens, dans leurs contenus stomacaux, leurs excréments, au sein de leurs organismes associés, ou encore dans les interactions (dont l'exploration est possible via de simples cooccurrences, ou par des changements de fréquences à travers le temps). Par exemple, une étude sur l'herbivorie réalisée à partir des parts d'herbier a permis de mettre en évidence les relations entre les taux d'herbivorie et les changements globaux (Meineke et Davies 2018; Meineke et al. 2019). Les changements environnementaux dans les océans sur plus de deux siècles peuvent être explorés grâce au cérumen des bouchons d'oreille des baleines bleues (voir remarque «Bouchons d'oreille des baleines: indicateurs de la qualité des océans » de ce chapitre). Ces recherches se basent toujours sur l'analyse des spécimens de collection, mais la perspective de trouver des indicateurs/marqueurs de l'environnement ouvre un univers entier d'utilisations très innovantes (les chapitres 6 et 11 à 16 en offrent plusieurs exemples). L'information sur les ensembles de spécimens (lots d'échantillons regroupés par date, récolteur et localité, le «event sampling » des anglophones) et leurs traitements de conservation qui leur ont été appliqués acquièrent une place importante dans ce type de recherche. L'accès à leurs états passés successifs et l'établissement de points de référence représentent ses principaux intérêts.

Le développement de la génétique, de la physiologie et de la biologie des populations contribuent aussi à l'utilisation des spécimens de collection pour des finalités qui n'avaient pas été envisagées lors de leur récolte. Dans certaines études, les collections sont utilisées pour répondre à des questions de restauration écologique, l'un des grands défis technologiques de notre époque. Par exemple, la réintroduction des individus produits à partir de graines et de spores conservées en herbier peut permettre la réintroduction d'allèles perdus et contribuer à l'enrichissement de la diversité génétique des petites populations (Nakahama et al. 2015). Les études dont le but est de faire revivre des espèces disparues en sont un autre exemple très spécifique. Comme Muller et al. l'ont bien montré (chapitre 10), la concrétisation de ce défi exige des expérimentations sur la physiologie de la germination et du développement sur des espèces proches afin de réduire l'impact sur le matériel cible, présent en petites quantités dans les collections. Le résultat final reste néanmoins très contraint par les conditions de conservation de ce matériel, qui n'avaient pas été prévu pour cette utilisation. Mais les connaissances acquises grâce aux études récentes nous permettent d'intervenir à la source du problème à l'heure de la conception de futures collections. Des nouveaux critères s'ajoutent pour le choix des espèces à inclure dans une banque de semences (par exemple espèces rares, en voie de disparition) et de nouvelles technologies sont 
développées pour préserver leur potentiel germinatif (Liu et al. 2018 ; Royal Botanic Gardens - Kew 2020).

\subsubsection{L'ère des données numériques}

La troisième sorte d'utilisations comprend des recherches basées sur les données virtuelles (transcription des informations des étiquettes, des cahiers de terrain, images, séquences moléculaires, sons, etc.) rendues disponibles par de nombreuses institutions sur des sites internet spécifiques ou à travers des portails agrégateurs. Elles ont en commun la focalisation sur la donnée et, trop fréquemment, un certain désintérêt vis-à-vis des spécimens ou des collections d'origine (Grandcolas 2017 ; chapitre 2 de ce volume), y compris quand leur origine est effectivement un spécimen (Troudet et al. 2018). Les impacts de cette démarche sont multiples : a) la perception de la taille colossale des lots de données sur la biodiversité disponibles via les collections (Page et al. 2015); b) la prise en compte de ces informations par des communautés scientifiques bien plus larges que celles qui sont familières des collections ; c) la possibilité d'étudier le vivant à des échelles spatio-temporelles sans précédent (Veron et al. 2019); d) la possibilité d'inclure des données de collection dans des protocoles divers. Néanmoins, ce Big Data est constitué par des milliards de données provenant d'une multitude de projets, chacun avec des objectifs, histoires, limites et méthodes propres. Comprendre ce que contiennent ces jeux de données, l'impact de notre ignorance sur leur complétude et leurs biais dans notre lecture du vivant est le grand défi d'aujourd'hui (Maldonado et al. 2015; Troudet et al. 2017 ; Daru et al. 2018 ; Dias Tarli et al. 2018). Cette nouvelle utilisation est en train de promouvoir de grandes transformations dans les analyses statistiques (Graham et al. 2004), dans les interactions entre les utilisateurs et dans toute la chaine de mise à disposition des données (voir chapitre 18). Le but est de pouvoir inclure le plus grand nombre possible dans les études, malgré leurs niveaux de précisions inégaux, les incertitudes sur leur exhaustivité et leurs différents biais. Plus récemment, ces données ont été également analysées avec le machine learning ou le e-learning qui cherchent des patrons au sein de leur diversité. Ces méthodes permettent ainsi l'exploration de problèmes qui nécessitent l'utilisation de grands lots de données (voir remarque «Estimer le niveau de menace de 300000 espèces de plantes », chapitre 18).

Ces deux dernières sortes d'utilisations témoignent de regards particulièrement innovants. Elles révèlent toute la puissance des approches basées sur de grands lots de données, même composites, ou pour la recherche d'informations présentes en quantités infimes dans les spécimens. Le spécimen n'est plus considéré seulement pour lui-même, mais comme un témoin d'une époque révolue et des changements 
environnementaux qui sont intervenus depuis. Cette utilisation est particulièrement importante à notre époque, en regard des changements globaux provoqués par les activités humaines.

\subsection{Les leçons de ces nouvelles utilisations}

Il est essentiel d'identifier et de comprendre les multiples regards que nous portons sur les collections de nos jours. Ils nous enseignent comment enrichir et gérer les collections afin qu'elles puissent offrir le maximum de leur contenu dans des approches futures que nous ne connaissons et n'imaginons pas forcément.

\subsubsection{L'importance de la richesse et de la diversité}

La première leçon apportée par cet ensemble de recherches innovantes concerne l'importance de la richesse et de la diversité des collections. Il est impossible de prévoir quelle espèce, quel spécimen, quel objet ou quelle information aura une importance cruciale pour la connaissance à court et à long terme. Mais les nouvelles utilisations nous montrent l'importance de l'immense réservoir où nous cherchons les informations pour tester nos hypothèses (voir remarque «Bouchons d'oreille des baleines : indicateurs de la qualité des océans » de ce chapitre). La diversité des collections représente de multiples options possibles pour les chercheurs aujourd'hui et dans le futur. Cette valeur d'option (Faith 2016 ; chapitre 20 de ce volume) est centrale à la contribution des collections aux approches scientifiques. Par conséquent, toutes les stratégies d'enrichissement et de conservation de collections devraient contribuer à améliorer tant la diversité que la qualité de l'échantillonnage. Se focaliser, par exemple, sur une espèce ou un groupe taxonomique d'intérêt ou, à l'inverse, n'incorporer que très peu d'exemplaires d'un maximum d'espèces sont des stratégies également peu propices à favoriser le nombre des options possibles. Comme pour la caractérisation de la diversité du vivant, la diversité phylogénétique (Faith 1992; Pellens et Grandcolas 2016) d'une collection peut être une mesure indirecte de sa valeur d'option (Liu et al. 2018).

\subsubsection{L'information au cœur des nouvelles recherches}

La deuxième leçon concerne l'information liée au spécimen, l'un des aspects qui a acquis une importance extrême pour les utilisations innovantes. Pour la systématique, un spécimen avec des informations de localité et de date de récolte très imprécises (par exemple : "Amérique septentrionale, $\mathrm{XVIII}^{\mathrm{e}}$ siècle ») - une situation fréquente pour les spécimens récoltés pendant le $\mathrm{XVIII}^{\mathrm{e}}$ et $\mathrm{XIX}^{\mathrm{e}}$ siècles - 
reste malgré tout exploitable tant que ses structures morpho-anatomiques et, dans le meilleur des cas, son ADN sont en bon état.

REMARQUE. Bouchons d'oreille des baleines : indicateurs de la qualité des océans

Les bouchons d'oreille des baleines sont composés d'une matrice de lipide et kératine, qui se dépose en forme de fines couches de couleur claire et sombre alternées. Ces couches s'accumulent semi-annuellement de manière systématique tout au long de la vie de l'individu et, pour cette raison, sont utilisées comme proxy pour déterminer l'âge des individus.

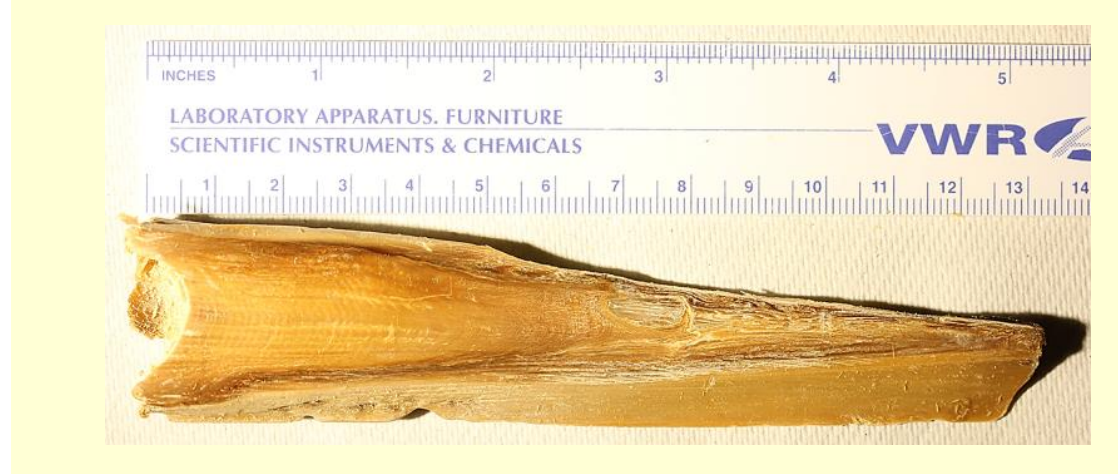

Figure 21.1. Section transversale d'un bouchon d'oreille d'un rorqual commun (Balaenoptera physalus). (C) Stephen J. Trumble, Baylor University, Waco, Texas, États-Unis.

COMMENTAIRE SUR LA FIGURE 21.1.- Les couches de cérumen claires et sombres alternées permettent de connaitre la composition chimique des lipides à chaque demiannée et représentent un indicateur de stress et de la composition chimique des océans pendant toute la vie de l'individu.

Des études récentes montrent que chacune de ces lames ont capturé des substances lipophiles (hormones et produits chimiques) représentatives des teneurs dans le corps de l'individu pour chaque demi-année de sa vie (Trumble et al. 2013). L'analyse des concentrations de cortisol (l'hormone du stress chez les mammifères), de mercure et de pesticides organiques dans ces lames permet ainsi d'évaluer les niveaux de stress et les concentrations de ces produits chimiques dans l'environnement où vivaient ces baleines (Trumble et al. 2018). Comme les baleines ont des aires de répartition très larges, vivent très longtemps (l'espérance de vie moyenne de plusieurs espèces est de l'ordre de 200 ans) et sont très 
sensibles aux changements de leur environnement, elles sont considérées comme des sentinelles des océans. Les informations recueillies dans les bouchons d'oreille se révèlent ainsi très utiles pour dater et marquer certains aspects des océans à une échelle planétaire et avec une profondeur temporelle sans précédent (des années 1870 à nos jours).

Pour de nombreuses utilisations innovantes, qu'elles soient liées aux caractéristiques des spécimens ou aux grands ensembles de données informatisées, les informations précises sur le spécimen, son lieu de vie ou sa date de récolte sont cruciales. Les spécimens avec des informations de récolte ou d'identification incomplètes ou peu précises ont une valeur réduite pour la plupart des utilisations. En outre, la qualité des informations n'est pas nécessairement garantie par l'usage d'outils technologiques nouveaux et puissants (par exemple géolocalisation, numérisation); la fiabilité et la pérennité de ces informations doit être absolument prise en compte.

\subsubsection{Bonne conservation et bonnes pratiques}

La troisième leçon concerne l'état et les moyens de conservation. Les techniques d'obtention d'information sur des spécimens s'améliorent et s'affinent de jour en jour. La capacité à mesurer des teneurs chimiques en quantités infimes est de plus en plus élevée ; les nouvelles technologies de séquençage permettent l'accès à l'ADN de presque tous les spécimens gardés dans les collections ; le CT-Scan (numérisation par tomographie aux rayons $\mathrm{X}$ assistée par ordinateur) permet l'examen de parties internes des spécimens sans recours à la dissection. En parallèle, les recherches sur les techniques de conservation s'intensifient et de nombreuses solutions sont mises à disposition (voir les nombreux exemples donnés par Véronique Rouchon, chapitre 20). Néanmoins, plus les spécimens peuvent être utilisés à des fins diverses, plus la bonne conservation et l'archivage des méthodes de conservation et des éventuels traitements sont nécessaires. Gaudeul et Rouhan (2013) l'ont souligné en ce qui concerne la conservation de l'ADN pour les études génétiques et moléculaires chez les plantes. Par exemple également, Peñuelas et Matamala (1990) ont pu constater le changement du contenu en azote et en souffre dans les feuilles de plantes vasculaires entre 1750 et 1990 en utilisant les spécimens d'une collection dont le traitement était connu et bien documenté ; elle avait été traitée au DDT, un pesticide que ne contient aucun de ces éléments, ce qui a permis d'exclure toute possibilité de contamination.

Un deuxième point relatif à la conservation est que les utilisations, qu'elles soient nouvelles ou anciennes, peuvent être invasives et/ou destructrices. Les effets de certaines utilisations sont bien connus, comme les prélèvements de tissus, le revêtement de structures avec de l'or pour la microscopie électronique à balayage, la 
préparation et le montage sur lames, l'insertion dans des résines, les colorations ((Espinosa et Pinedo Castro 2018) ; Rouchon dans le chapitre 20). Mais d'autres utilisations ont des impacts plus subtils, invisibles à l'œil nu, voire variables selon les organismes ou le type de structure. Il faut donc leur accorder une attention particulière pour s'assurer de la bonne conservation des spécimens et de leurs utilisations ultérieures. Par exemple, la tomographie aux rayons X (CT-Scan) peut endommager l'ADN des tissus durs minéralisés (os, dents) tandis que son impact direct sur les tissus mous reste mal connu. En outre, cette technologie appliquée aux tissus mous requiert l'utilisation des produits contrastants, aux caractéristiques diverses et aux effets spécifiques (Hall et al. 2015).

Pour assurer la conservation et l'intégrité des spécimens, il est donc important de détecter les effets possibles des différentes méthodes et de mettre à disposition des guides de bonnes pratiques afin d'atténuer leurs impacts (comme le guide produit par (Keklikoglou et al. 2019)). Des groupes de travail ad hoc et la constitution de groupes d'experts recensant les nouvelles utilisations et leurs impacts sur les spécimens à différentes échelles de temps sont aussi très souhaitables. Les consignes en la matière sont d'autant plus importantes que le nombre d'utilisations potentielles augmente, surtout avec l'informatisation des collections et la disponibilité des informations acquises sur les spécimens (codes-barres, séquences, isotopes, images diverses 2D, 3D - voir chapitre 17). Dans ce contexte, les spécimens les mieux étudiés auront tendance à être repris plus souvent pour d'autres recherches, qui peuvent s'appuyer sur certaines des connaissances déjà acquises.

De nos jours, une autre facette de ce sujet concerne la conservation des données. Ainsi qu'il est signalé dans le chapitre 18 (Monnet et al.), l'informatisation est un enjeu important qui relie toute la chaine de l'acquisition à l'utilisation finale des données. Comme tout ce qui est lié à l'informatique, ce sujet évolue très rapidement. De nombreuses institutions veillent à que les données restent bien à disposition dans l'avenir, suivant la définition des principes FAIR (Findable, Accessible, Interoperable and Reusable) ${ }^{1}$. À ce titre, il est primordial que les données soient enregistrées dans des formats et sur des supports qui résistent à l'obsolescence informatique et aux crashs des grands systèmes opérationnels. Des fichiers au format .txt, .rtf, .csv, qui peuvent être ouverts par une très large gamme de logiciels, et des dépôts open source des données et des métadonnées peuvent contribuer à la pérennité des informations.

\footnotetext{
${ }^{1}$ https://www.go-fair.org/fair-principles.
} 


\subsubsection{De l'importance des ensembles}

Les recherches des informations sur l'environnement passé, sur les impacts des changements environnementaux sur les organismes (interactions, phénologie, etc.) à partir des spécimens de collection met en avant l'importance des ensembles (event sampling en anglais). C'est une nouvelle dimension qui vient s'ajouter au débat récurrent sur le rangement idéal des spécimens dans les collections. Des lots de spécimens issus d'un même évènement de récolte peuvent représenter, dans leur ensemble, des échantillons ou des réplicats d'une interaction ou d'une caractéristique environnementale à des lieux et des dates connus (GBIF 2017).

La dispersion des ensembles dans plusieurs unités de rangement réduit fortement ce potentiel car elle génère des pertes d'informations. Même dans une situation où tous les spécimens seraient totalement informatisés et facilement réassociés, ce qui est le cas pour une minorité de groupes taxonomiques dans les collections, certaines informations non informatisées a priori peuvent échapper au regard tout simplement parce que les individus ne sont pas regroupés. Comme par exemple s'apercevoir que des échantillons récoltés plus tard dans l'année ne présentent pas de traces de consommation par des herbivores. Les ensembles offrent également des opportunités de mise en place de séries temporelles permettant de «monitorer » et de comprendre les changements de l'environnement sur le long terme.

Il est bien évident que la gestion des collections est contrainte par des problèmes logistiques divers et qu'un rangement taxonomique est indissociable de l'existence des collections. Néanmoins, dans le cas où cette option serait possible, il ne faut surtout pas assumer, a priori, que toutes les informations résidentes dans des ensembles pourraient être récupérées avec l'informatisation. Pour cela, le cahier des charges de l'informatisation devrait inclure un éventail d'informations à prendre en compte bien plus large que celui pratiqué couramment.

\subsection{Les collections dans la science du $x \mathrm{XI}^{\mathrm{e}}$ siècle et au-delà}

Cette diversité de nouvelles utilisations nous montre combien la richesse en informations est centrale à la valeur d'option des collections. De ce fait, de nouvelles responsabilités incombent aux récolteurs d'aujourd'hui. Les collectes traditionnelles avec les seules dates et lieux de récolte sont ainsi devenues bien limitées en regard du savoir-faire scientifique qui s'est développé dans ce domaine (Schindel et Cook 2018). Nous avons les moyens de documenter tout ce qui peut être vu sur le spécimen à travers des images numériques $2 \mathrm{D}, 3 \mathrm{D}$ ou même $4 \mathrm{D}$ (chapitre 17). Des informations sur les comportements, les interactions, les détails des micro-habitats, ainsi que la récolte des espèces associées (prédateurs, proies, 
plantes hôtes, endosymbiotes, etc.) sont également précieuses. La prise en compte de la diversité à tous les niveaux d'observation doit être encouragée; plusieurs spécimens d'une espèce en regard de la diversité intraspécifique, plusieurs prélèvements sur un même individu pour comprendre la diversité génétique et phénotypique intra-organisme (par exemple des parts d'herbiers correspondant à des prélèvements localisés à différentes hauteurs dans un arbre) et ainsi de suite. Cette conception, que certains appellent le spécimen étendu (de l'anglais «the extended specimen ») est maintenant devenue indissociable de l'histoire naturelle.

Notre responsabilité lors des nouvelles collectes est d'autant plus importante que nous traversons une crise majeure de la biodiversité. Pour faire face à cette crise, nous avons besoin d'informations sur les espèces à faibles effectifs populationnels (rares ou endémiques) qui sont fréquemment vulnérables à l'extinction. Dans ce contexte où de multiples transformations irréversibles de l'environnement ont lieu chaque année, les évènements de collecte représentent des opportunités uniques de garder les traces d'un patrimoine pour les générations qui nous succèderont. Bien évidemment, cette situation de crise nous incite également à plus de rigueur dans le partage des avantages (APA) et les autres questions éthiques liées aux utilisations de collections. La disponibilité des collections et des informations résidentes ne relève pas seulement d'une bonne déontologie scientifique mais aussi du respect des accords politiques établis entre différentes parties au plan international. C'est le cadre, par exemple, du protocole de Nagoya pour la biodiversité ou des accords de restitutions pour les restes ou artefacts humains.

\subsection{Pour conclure}

Nous pouvons conclure cet ouvrage en nous posant la question du rôle des collections dans les sciences du futur. Pouvons-nous envisager des sciences du vivant sans les collections? Pouvons-nous envisager une relation harmonieuse entre l'Homme et la nature sans les connaissances basées sur les collections ?

Connaitre le vivant et l'Homme représente essentiellement le défi d'intégrer leur diversité, ce produit incroyable de l'évolution de la vie. Néanmoins, notre esprit a tendance à écarter tout ce qui n'est pas déjà connu (Kuhn 2012). Les collections représentent un outil fabuleux pour contrer ce biais de confirmation et combattre notre anthropocentrisme naturel en prenant conscience de la diversité. Par conséquent, le premier risque de sciences du vivant sans collection est celui de se focaliser sur l'espèce humaine et quelques organismes modèles en faisant l'impasse sur une diversité indispensable à la compréhension d'enjeux essentiels, humains ou environnementaux. 
Ne pas avoir ou perdre des collections peut conduire à une deuxième extinction, c'est-à-dire à effacer de notre mémoire l'existence de cette énorme diversité que notre génération perçoit encore (Raven et Miller 2020). L'histoire récente nous montre que certaines espèces, auparavant largement répandues avec d'énormes populations, comme par exemple le pigeon migrateur en Amérique du Nord, se sont éteintes en quelques décennies. Dans le même intervalle de temps réduit, de vastes forêts ont été remplacées par des mégapoles et seules les collections permettent encore de rendre compte de la diversité qui y existait. L'incendie qui a détruit le Museu Nacional de Rio de Janeiro en septembre 2018 est un exemple tragique de ce genre de perte. Il n'a pas seulement effacé une grande partie des types des espèces d'organismes (en particulier les insectes) ou d'artefacts décrits au Brésil au cours du $\mathrm{XX}^{\mathrm{e}}$ siècle. Avec l'incendie, nous avons perdu les témoins de la diversité d'une région tropicale extrêmement riche et qui n'existe plus (Pellens et Grandcolas 2018).

Les collections sont également une extraordinaire source d'inspiration pour chercher des solutions à de multiples questions ou problèmes. Le domaine de la bioinspiration ou des solutions fondées sur la nature, central pour nos sociétés industrielles, en dépend fortement pour son plein redéploiement (Benyus 1997; Chapelle et Raskin 2020). Les perdre ou ne pas en disposer nous priverait d'un outil essentiel pour cette inspiration et ce regard sur le vivant.

Plus généralement, les collections sont un exemple précurseur et pourtant bien vivant de la science ouverte, dans laquelle les résultats mais aussi les données de la recherche devraient être disponibles à tout un chacun (Schilthuizen et al. 2015). Elles doivent continuer à inspirer l'ensemble de la science dans l'application d'une politique de science ouverte, en allant plus loin que d'assurer une simple disponibilité électronique trop souvent assimilée à l'ouverture des données.

Enfin, cette ouverture doit être aussi celle vers le public et la société civile, bien au-delà des frontières, d'ailleurs imaginaires, de la communauté scientifique (Bakker et al. 2020). Les collections doivent également être un outil pour aider à la pédagogie, à la muséographie et à toutes les autres disciplines liées à la connaissance au sein de nos sociétés.

\subsection{Bibliographie}

Bakker, F.T., Antonelli, A., Clarke, J.A., Cook, J.A., Edwards, S.V, Ericson, P.G.P., Faurby, S., Ferrand, N. (2020). The Global Museum: natural history collections and the future of evolutionary science and public education. PeerJ, 2020, 1-40.

Benyus, J.M. (1997). Biomimicry: Innovation inspired by nature. William Morrow, New York. 
Chapelle, G., Raskin, K. (dir.) (2020). Humanité bio-inspirée; une autre approche. Le Cherche Midi, Cité des Sciences et de l'Industrie, Paris.

Daru, B.H., Park, D.S., Primack, R.B., Willis, C.G., Barrington, D.S., Whitfeld, T.J.S., Seidler, T.G., Sweeney, P.W., Foster, D.R., Ellison, A.M., Davis, C.C. (2018). Widespread sampling biases in herbaria revealed from large-scale digitization. New Phytologist, 217(2), 939-955.

Darwin, C. (1845). Journal of Researches into the Natural History and Geology of the Countries Visited During the Voyage of H.M.S. Beagle Round the World, under the Command of Capt. Fitz Roy, R.N., $2^{\mathrm{e}}$ édition. John Murray, Londres.

Darwin, C. (1859). On the Origin of Species by Means of Natural Selection, or the Preservation of Favoured Races in the Struggle for Life. John Murray, Londres.

Dias Tarli, V., Grandcolas, P., Pellens, R. (2018). The informative value of museum collections for ecology and conservation: A comparison with target sampling in the Brazilian Atlantic forest. PLoS ONE, 13, e0205710.

Espinosa, F., Pinedo Castro, M. (2018). On the use of herbarium specimens for morphological and anatomical research [En ligne]. Botany Letters, 165(3-4), 361-367. Disponible à l'adresse : http://doi.org/10.1080/23818107.2018.1451775.

Faith, D.P. (1992). Conservation evaluation and phylogentic diversity. Biological Conservation, 61, 1-10.

Faith, D.P. (2016). A general model for biodiversity and its value. Dans The Routledge Handbook of Philosophy of Biodiversity, Garson, J., Plutynski, A., Sarkar, S. (dir.). Routledge, Abingdon-on-Thames, 69-85.

Gaudeul, M., Rouhan, G. (2013). A plea for modern botanical collections to include DNAfriendly material. Trends in Plant Science, 18(4), 184-185.

GBIF (2017). Best Practices in Publishing Sampling-event data [En ligne]. Disponible à l'adresse : https://github.com/gbif/ipt/wiki/BestPracticesSamplingEventData.

Giribet, G. (2010). A new dimension in combining data? The use of morphology and phylogenomic data in metazoan systematics. Acta Zoologica, 91(1), 11-19.

Graham, C.H., Ferrier, S., Huettman, F., Moritz, C., Peterson, A.T. (2004). New developments in museum-based informatics and applications in biodiversity analysis. Trends in Ecology and Evolution, 19(9), 497-503.

Grandcolas, P. (2017). Losing the connection between the observation and the specimen: a by-product of the digital era or a trend inherited from general biology?. Bionomina, 12, $57-62$.

Hall, A.C., Sherlock, E., Sykes, D. (2015). Does Micro-CT scanning damage DNA in museum specimens. Journal of Natural Science Collections, 2, 22-28.

Keklikoglou, K., Faulwetter, S., Chatzinikolaou, E., Wils, P., Brecko, J., Kvaček, J., Metscher, B., Arvanitidis, C. (2019). Micro-computed tomography for natural history specimens: a handbook of best practice protocols. European Journal of Taxonomy, 1-55. 
Kuhn, T.S. (2012). The structure of scientific revolutions. University of Chicago Press, Chicago.

Liu, U., Breman, E., Cossu, T.A., Kenney, S. (2018). The conservation value of germplasm stored at the Millennium Seed Bank, Royal Botanic Gardens [En ligne]. Biodiversity and Conservation, 27(6), 1347-1386. Disponible à l'adresse : https://doi.org/10.1007/s10531018-1497-y.

Maldonado, C., Molina, C.I., Zizka, A., Persson, C., Taylor, C.M., Albán, J., Chilquillo, E., Rønsted, N., Antonelli, A. (2015). Estimating species diversity and distribution in the era of Big Data: To what extent can we trust public databases?. Global Ecology and Biogeography, 24(8), 973-984.

Meineke, E.K., Davies, T.J. (2018). Museum specimens provide novel insights into changing plant - herbivore interactions. Philosophical Transactions of the Royal Society of London, B, 374(2017.0393).

Meineke, E.K., Classen, A.T., Sanders, N.J., Davies, T.J. (2019). Herbarium specimens reveal increasing herbivory over the past century. Journal of Ecology, 107(juillet 2018), 105117.

Nakahama, N., Hirasawa, Y., Minato, T., Hasegawa, M., Isagi, Y., Shiga, T. (2015). Recovery of genetic diversity in threatened plants through use of germinated seeds from herbarium specimens. Plant Ecology, 2016, 1635-1647.

Page, L.M., Macfadden, B.J., Fortes, J.A., Soltis, P.S., Riccardi, G. (2015). Digitization of Biodiversity Collections Reveals Biggest Data on Biodiversity. BioScience, 65(9), 841842.

Pellens, R., Grandcolas, P. (dir.) (2016). Biodiversity Conservation and Phylogenetic Systematics: preserving our evolutionary heritage in an extinction crisis [En ligne]. Springer, Cham. Disponible à l'adresse: http://link.springer.com/10.1007/978-3-31922461-9.

Pellens, R., Grandcolas, P. (2018). Après l'incendie du Museu Nacional de Rio : que faire maintenant? [En ligne]. The Conversation. Disponible à l'adresse : $\mathrm{http}: / /$ theconversation.com/apres-lincendie-du-museu-nacional-de-rio-que-fairemaintenant-104115.

Peñuelas, J., Matamala, R. (1990). Changes in N and S leaf content, stomatal density and specific leaf area of 14 plant species during the last three centuries of $\mathrm{CO}_{2}$ increase. Journal of Experimental Botany, 41(9), 1119-1124.

Petzold, A., Hassanin, A. (2020). A comparative approach for species delimitation based on multiple methods of multi-locus DNA sequence analysis: A case study of the genus Giraffa (Mammalia, Cetartiodactyla). PLoS ONE, 2020, 1-28.

Petzold, A., Magnant, A., Edderai, D., Chardonnet, B., Rigoulet, J., Saint-Jalme, M., Hassanin, A. (2020). First insights into past biodiversity of giraffes based on mitochondrial sequences from museum specimens. European Journal of Taxonomy, 703, $1-33$. 
Raven, P.H., Miller, S. (2020). Here today, gone tomorrow. Science, 370(6513), 149.

Royal Botanic Gardens - Kew (2020). A Global Resource for Plant and Fungal Knowledge: Science strategy 2015-2020. Rapport, ORBIS.

Schindel, D.E., Cook, J.A. (2018). The next generation of natural history collections [En ligne]. PLoS Biology, 2018, 1-8. Disponible à l'adresse: doi.org/10.1371/ journal.pbio.2006125.

Schilthuizen, M., Vairappan, C.S., Slade, E.M., Mann, D.J., Miller, J.A. (2015). Specimens as primary data: museums and 'open science'. Trends in Ecology \& Evolution, 30, 237-238.

Schubnel, T., Desutter-Grandcolas, L., Legendre, F., Prokop, J., Mazurier, A., Garrouste, R., Grandcolas, P., Nel, A. (2020). To be or not to be: postcubital vein in insects revealed by microtomography. Systematic Entomology, 45(2), 327-336.

Troudet, J., Grandcolas, P., Blin, A., Vignes-Lebbe, R., Legendre, F. (2017). Taxonomic bias in biodiversity data and societal preferences. Scientific Reports, 7(1), 1-14.

Troudet J., Vignes-Lebbe, R., Grandcolas, P., Legendre, F. (2018). The Increasing Disconnection of Primary Biodiversity Data from Specimens: How Does It Happen and How to Handle It?. Systematic Biology, 67, 1110-1119.

Trumble, S.J., Robinson, E.M., Berman-Kowalewski, M., Potter, C.W., Usenko, S. (2013). Blue whale earplug reveals lifetime contaminant exposure and hormone profiles. Proceedings of the National Academy of Sciences of the United States of America, 110(42), 16922-16926.

Trumble, S.J., Norman, S.A., Crain, D.D., Mansouri, F., Winfield, Z.C., Sabin, R., Potter, C.W., Gabriele, C.M., Usenko, S. (2018). Baleen whale cortisol levels reveal a physiological response to $20^{\text {th }}$ century whaling. Nature Communications, 9(1).

Veron, S., Haevermans, T., Govaerts, R., Mouchet, M., Pellens, R. (2019). Distribution and relative age of endemism across islands worldwide [En ligne]. Scientific Reports, 9(1), 11693. Disponible à l'adresse : https://doi.org/10.1038/s41598-019-47951-6. 